\title{
Two New Criteria of the Metabolic Syndrome: Prevalence and the Association with Brachial-Ankle Pulse Wave Velocity in Japanese Male Workers
}

\author{
Koichi MiYaki ${ }^{1,2}$, Asako Hara ${ }^{1}$, Mariko Naito ${ }^{3}$, Toru Naito ${ }^{4}$ and Takeo NaKayama ${ }^{5}$
}

${ }^{1}$ Department of Preventive Medicine and Public Health, School of Medicine, Keio University, ${ }^{2}$ Department of Molecular Epidemiology, Medical Research Institute, Tokyo Medical \& Dental University, ${ }^{3}$ Department of Preventive Medicine/Biostatictics and Medical Decision Making, Nagoya University Graduate School of Medicine, ${ }^{4}$ Section of General Dentistry, Department of General Dentistry, Fukuoka Dental College and ${ }^{5}$ Department of Health Informatics, Kyoto University School of Public Health, Japan

\begin{abstract}
Two New Criteria of the Metabolic Syndrome: Prevalence and the Association with Brachial-Ankle Pulse Wave Velocity in Japanese Male Workers: Koichi MiYAKI, et al. Department of Preventive Medicine and Public Health, School of Medicine, Keio University-In 1998 and 2001, The World Health Organization and the National Cholesterol Education Program Adult Treatment Panel III proposed working criteria for the metabolic syndrome (MS), but they are not perfect for use in diverse ethnicities. In 2005, the International Diabetes Federation (IDF) and eight societies in Japan respectively proposed new criteria. However, there has been no report regarding the application of these new criteria in Japanese workplaces. We conducted a cross-sectional study of 377 healthy Japanese men aged 20-64 yr who worked in a chemical factory in Kanagawa, Japan. Participants completed a self-reported questionnaire, underwent a physical examination including waist measurements and brachial-ankle pulse wave velocity (baPWV), and provided overnight fasting blood samples. The prevalence of MS in Japanese men was $17.0 \%$ and $13.5 \%$ according to the new IDF and Japanese criteria respectively. In both of the new criteria, baPWV was significantly higher in those with MS than those without MS $(1,563 \pm 264.2$ vs $1,362 \pm 204.6 \mathrm{~cm} / \mathrm{sec}, p<0.001$ in the new IDF criterion; $1,574 \pm 265.2$ vs $1,368 \pm 209.1$ $\mathrm{cm} / \mathrm{sec}, p<0.001$ in the Japanese criterion). In the analysis of the 5 or 6 subgroups stratified according to the number of MS components, baPWV increased significantly with increasing number of MS components
\end{abstract}

Received Aug 23, 2005; Accepted Jan 10, 2006

Correspondence to: K. Miyaki, Department of Preventive Medicine and Public Health, School of Medicine, Keio University, 35 Shinanomachi, Shinjuku-ku, Tokyo 160-8582, Japan

(e-mail:miyaki@sc.itc.keio.ac.jp.)
( $p$ for trend $<0.01$ in both criteria). The new IDF and Japanese criterion are both good for diagnosing MS among Japanese because a linear increase in baPWV occurred with increasing MS components after adjustment for potential confounding factors. Further studies are expected using these new criteria.

(J Occup Health 2006; 48: 134-140)

Key words: Metabolic syndrome, baPWV, New criteria, Japan

Metabolic syndrome (MS) is a cluster of metabolic disorders including central obesity, glucose intolerance, hypertension, and dyslipidemia ${ }^{1,2)}$. These traits occur simultaneously to a greater degree than would be expected by chance alone $e^{3,4)}$. Although the contribution of an individual trait may be small, multiple traits may act together and exert an even greater influence than any one trait alone ${ }^{5)}$.

The concept of MS has become well established, and several studies have suggested that subjects with MS are at an increased risk for type 2 diabetes ${ }^{6,7)}$ and cardiovascular disease ${ }^{8-12}$. However, there has been no uniform case definition for MS.

In 1998 and 2001, the World Health Organization $(\mathrm{WHO})^{13)}$ and the National Cholesterol Education Program Adult Treatment Panel III (NCEP ATP III) ${ }^{14)}$ each proposed working criteria for MS. The NCEP ATP III report recommended the use of five variables for diagnosis of MS, including waist circumference, triglyceride level, HDL cholesterol level, blood pressure, and fasting glucose level. Subjects meeting three of these five criteria are classified as having MS. The WHO criteria are more complex because they take account of microalbuminuria and plasma insulin levels. 
According to the NCEP ATP III and WHO criteria, several studies of the prevalence and characteristics of MS have been conducted among diverse ethnicities ${ }^{15-23)}$. Some studies showed ethnic variation in the prevalence of $\mathrm{MS}^{15,16,23)}$ and some studies which conducted in Asia suggested that the definition of central obesity recommended by the NCEP ATP III was inappropriate for Asian populations which are generally of smaller build than Caucasians because the criterion had been based on data from Caucasian cohorts ${ }^{16,17,21)}$.

In consideration of the ethnic differences in prevalence and characteristics of MS, the International Diabetes Federation (IDF) proposed the worldwide MS criteria that includes a criterion for Japanese in April 2005 ${ }^{24)}$. According to the new IDF criteria, a person defined as having MS must have central obesity plus any two of the following four factors: 1) raised triglyceride level; 2) reduced HDL cholesterol; 3) raised blood pressure; 4) raised fasting plasma glucose. With regard to the definition of central obesity, ethnic specific values for waist circumference are used.

At almost the same time, in April of 2005, eight societies in Japan, the Japan Society for the Study of Obesity, the Japan Atherosclerosis Society, the Japan Diabetes Society, the Japanese Society of Hypertension, the Japanese Circulation Society, the Japanese Society of Nephrology, the Japanese Society on Thrombosis and Hemostasis, and the Japanese Society of Internal Medicine, proposed a new criterion of MS for Japanese ${ }^{25}$. According to the criterion, subjects are defined as having MS if they have central obesity plus any two of the following three factors: 1) dyslipidemia (raised TG level and/or reduced HDL cholesterol); 2) raised blood pressure; 3) raised fasting plasma glucose. To our knowledge no assessment of these new criteria has been performed among Japanese workers.

Pulse wave velocity (PWV) is an index of arterial stiffness $^{26,27)}$, and is regarded as a non-invasive marker of vascular damage ${ }^{28-30)}$. Previous studies have demonstrated that PWV is a marker of the severity of cardiovascular disease $^{31)}$ and a predictor of future events ${ }^{32-34)}$. As well, it is applicable as a screening tool for cardiovascular risk in a general population ${ }^{29,35)}$. Recently, a simple device for measuring brachial-ankle PWV (baPWV) has been developed and made available ${ }^{36,37)}$.

MS is a predictor of cardiovascular mortality ${ }^{8-10)}$ and many studies have focused on the association of cardiovascular disease and its risk factors with increased PWV 31, 32, 34, 36-38). However, there are almost no studies relating PWV to $\mathrm{MS}^{5,39)}$, and no study to our knowledge has investigated the relationship between PWV and MS as defined by the new criteria.

The aims of this study were to assess the prevalence and characteristics of MS using the two new criteria, the new IDF criterion and the Japanese criterion, and to investigate the association between baPWV and MS in middle-aged Japanese men.

\section{Methods}

We conducted a cross-sectional study of Japanese male workers who worked in a chemical factory in Kanagawa, Japan. Of the 438 male workers, 377 (86.1\%) aged 2064 yr participated in the study. We divided the subjects into two subgroups according to the kind of occupation, office workers and laborers. Office workers were 176 $(46.7 \%)$ and laborers were $201(53.3 \%)$.

Of 377 subjects, 43 (11.4\%), 4 (1.1\%), and 7 (1.9\%) were taking medication for hypertension, dyslipidemia, and diabetes, respectively. Although there were the potential effects of medications on metabolic values, we did not exclude the above-mentioned subjects because they should be included in each criterion of MS and for the purpose of reflecting the workplace population as it is without selection bias.

Participants completed a self-reported questionnaire (including medical history, medication use and smoking status), underwent a physical examination (including height, weight, waist circumference, blood pressure, and baPWV measurements), and provided overnight fasting blood samples. Body mass index (BMI) was calculated by $\mathrm{kg} / \mathrm{m}^{2}$. baPWV was measured by well-trained nurses on the left and right sides using the PWV/ABI device (Nippon Colin, Aichi, Japan) which was approved by the US Food and Drug Administration (FDA) as VP-2000/ 1000. We used the mean value of the right and left baPWV values. Of 377 subjects, 67 (17.8\%) showed $\geq 1,600 \mathrm{~cm} / \mathrm{sec}$ baPWV and were diagnosed as having abnormally high PWV values. In this study, past smokers and those who had never smoked were combined and compared with current smokers. All subjects in the study gave their informed consent for the use of personal information for analysis. The Ethics Committee of the Keio University School of Medicine, Tokyo, Japan, approved the study protocol.

\section{Definition of metabolic syndrome}

According to the new IDF criterion, the characteristics of MS in Japan are defined by the following cutoff limits and the subjects are defined as having MS if they have central obesity plus two or more of the other components: 1) central obesity (waist circumference $\geq 85 \mathrm{~cm}$ in men); 2) raised triglyceride level (triglyceride $\geq 150 \mathrm{mg} / \mathrm{d} l$ ) or specific treatment for this lipid abnormality; 3) reduced HDL cholesterol (HDL cholesterol $<40 \mathrm{mg} / \mathrm{d} l$ ) or specific treatment for this lipid abnormality; 4) raised blood pressure (systolic blood pressure $\geq 130 \mathrm{mmHg}$ or/and diastolic blood pressure $\geq 85 \mathrm{mmHg}$ ) or treatment of previously diagnosed hypertension; 5) raised fasting plasma glucose (fasting plasma glucose $\geq 100 \mathrm{mg} / \mathrm{d} l$ ) or previously diagnosed type 2 diabetes. 
According to the Japanese criterion, the characteristics of MS are defined by the following cutoff limits and the subjects are defined as having MS if they have central obesity plus two or more of the other components: 1) central obesity (waist circumference $\geq 85 \mathrm{~cm}$ in men); 2 ) dyslipidemia (triglyceride $\geq 150 \mathrm{mg} / \mathrm{d} l$ or/and HDL cholesterol $<40 \mathrm{mg} / \mathrm{d} l$ ) or medication for dyslipidemia; 3 ) raised blood pressure (systolic blood pressure $\geq 130$ $\mathrm{mmHg}$ or/and diastolic blood pressure $\geq 85 \mathrm{mmHg}$ ) or medication for hypertension; 4) raised fasting plasma glucose (fasting plasma glucose $\geq 110 \mathrm{mg} / \mathrm{d} l$ ) or medication for diabetes.

According to the NCEP ATP III criterion ${ }^{14)}$, the characteristics of MS in men are defined by the following cutoff limits and subjects having three or more of the components are defined as having MS: 1) waist circumference $>102 \mathrm{~cm}$; 2) triglyceride $\geq 150 \mathrm{mg} / \mathrm{d} l$; 3) HDL cholesterol $<40 \mathrm{mg} / \mathrm{d} l$; 4) systolic blood pressure $\geq 130 \mathrm{mmHg}$ or/and diastolic blood pressure $\geq 85 \mathrm{mmHg}$ or known treatment for hypertension; 5) fasting plasma glucose $\geq 110 \mathrm{mg} / \mathrm{d} l$ or known treatment for diabetes.
Statistical analysis

All log-normally distributed variables (fasting plasma glucose, triglyceride, and HDL cholesterol) were logtransformed before statistical analysis and backtransformed for reporting. Differences in metabolic, anthropometric, and numerical demographic variables between individuals with and without MS were assessed using independent samples $t$ testing. The $\chi^{2}$ test was used to determine whether frequencies for categorical variables differed between these two groups of subjects. Age-, BMI-, systolic blood pressure (SBP)-, and smoking statusadjusted Pearson correlations were calculated to investigate the relationship between baPWV and MS as well as other metabolic and anthropometric variables. Multivariate logistic regression analysis was used to assess the relationship between risk of increased baPWV and each feature of MS. All analyses were performed with the SPSS statistical package for Windows version 12.0. All reported $\mathrm{p}$-values were two-sided, and $p<0.05$ was considered statistically significant.

Table 1. Clinical and biochemical characteristics of all the subjects and the subjects with or without metabolic syndrome by the Japanese criterion and the new IDF criterion

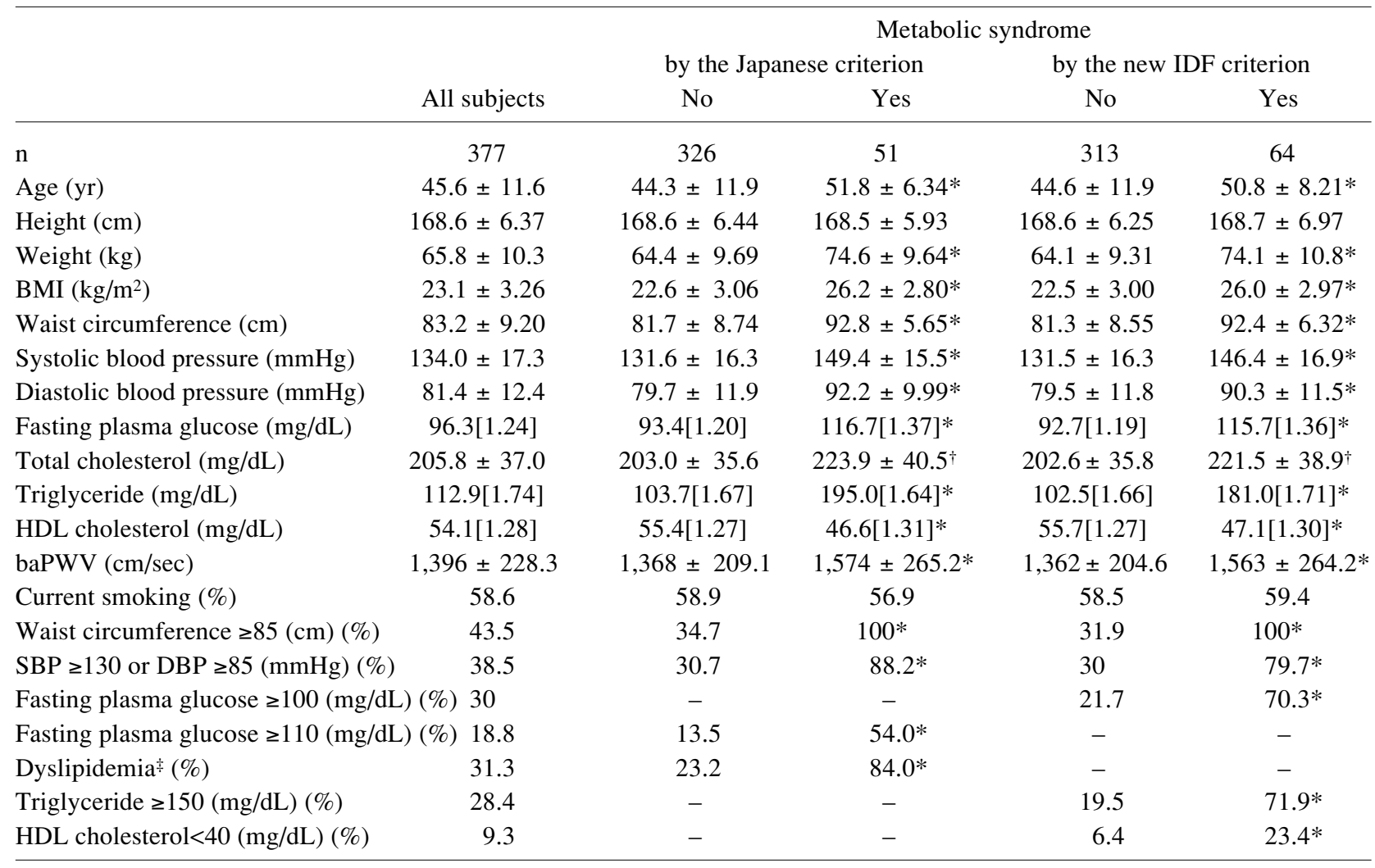

Data are means \pm SD and $\%$ for normally distributed variables, and geometric means [GSD] for non-normally distributed variables. ${ }^{*} p<0.001 ;{ }^{\dagger} p=0.001$ for the $\mathrm{t}$ test of differences in means or the $\chi^{2}$ test of differences in proportions for continuous and categorical variables, respectively, in comparisons between subjects with or without metabolic syndrome.

†riglyceride $\geq 150(\mathrm{mg} / \mathrm{dL})$ or/and HDL cholesterol $<40(\mathrm{mg} / \mathrm{dL})$. 

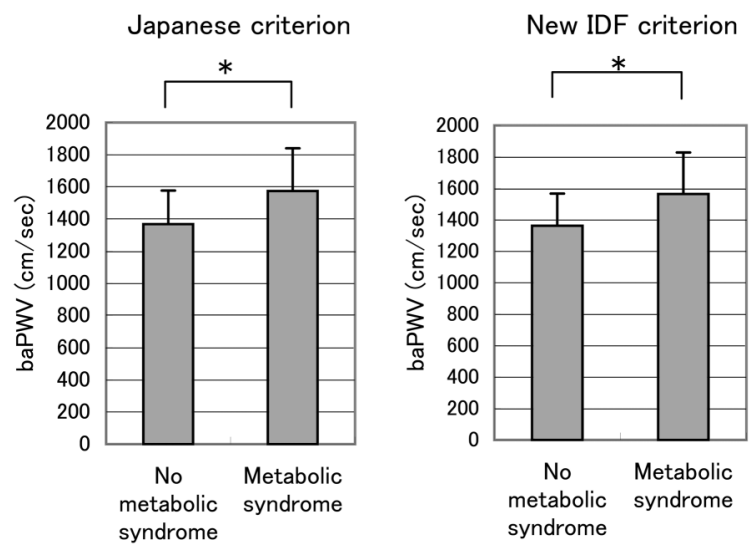

Fig. 1. The relation between baPWV values and metabolic syndrome by the Japanese criterion and the new IDF criterion. ${ }^{*} p<0.001$ for the $t$ test of differences in means in comparisons between subjects with or without metabolic syndrome.
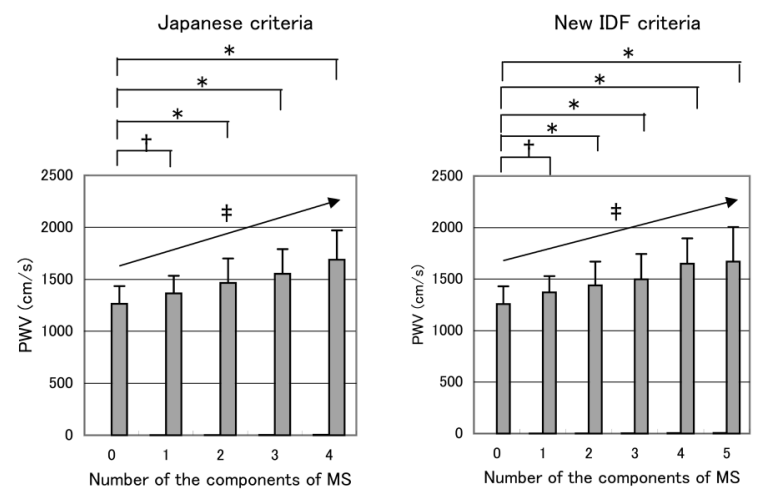

Fig. 2. The relation between baPWV values and the number of components of metabolic syndrome by the Japanese criterion and the new IDF criterion. $* p<0.001 ; \uparrow<0.01$ for Tukey’s multiple comparison test. $₫ p<0.01$ for linear trend test adjusted for age, BMI, SBP and smoking status.

Table 2. Multivariate logistic regression analysis with baPWV* as a dependent variable and metabolic syndrome as an indepedent variable in the Japanese criterion and the new IDF criterion

\begin{tabular}{lcc}
\hline & OR $(95 \% \mathrm{CI})^{\dagger}$ & $p$ \\
\hline Metabolic syndrome by the Japanese criterion & $2.08(0.90-4.81)$ & 0.087 \\
Metabolic syndrome by the new IDF criterion & $2.88(1.30-6.37)$ & 0.009 \\
\hline
\end{tabular}

*High baPWV was defined as $\geq 1,600 \mathrm{~cm} / \mathrm{sec}$, †Adjusted for age, BMI, Systolic blood pressure (SBP), and smoking status.

\section{Results}

Table 1 shows the clinical and biochemical characteristics of all the subjects and the subjects with or without MS by the new IDF criterion and the Japanese criterion. The prevalence of MS was $17.0 \%(n=64)$ in the new IDF criterion and $13.5 \%(n=51)$ in the Japanese criterion. Using the NCEP ATP III criterion, the prevalence of MS was 5.84\% (data not shown).

In both the new criteria, subjects with MS were significantly older, and had higher BMI, waist circumference, SBP, DBP, fasting plasma glucose, triglyceride, and baPWV but smaller HDL cholesterol than the subjects without MS. Height and percentage of current smoking subjects did not differ between the two groups in both the criteria.

In the analysis of two subgroups, laborers and office workers, the prevalences of MS were not significantly different in both the new IDF and Japanese criteria ( $p=0.083$ in the IDF criterion; $p=0.111$ in the Japanese criterion) (data not shown).

baPWV was significantly higher in those with MS than those without MS in both the criteria $(1,563 \pm 264.2$ vs
$1,362 \pm 204.6 \mathrm{~cm} / \mathrm{sec}, p<0.001$ in the new IDF criterion; $1,574 \pm 265.2$ vs $1,368 \pm 209.1 \mathrm{~cm} / \mathrm{sec}, p<0.001$ in the Japanese criterion) (Fig. 1). After adjustment for age, BMI, SBP, and smoking status, baPWV was still significantly higher in those with MS than those without MS ( $p=0.001$ in the new IDF criterion; $p=0.045$ in the Japanese criterion). In the two subgroups, laborers and office workers, baPWV was also significantly higher in those with MS than those without MS in both the criteria $(p<0.001$ in both the criteria in both subgroups) (data not shown).

Subjects with different numbers of components of MS were placed in 6 and 5 subgroups, respectively, in the new IDF criterion and the Japanese criterion (graded from 0 through 5 and 4, respectively). Following stratification into the 6 and 5 subgroups, age-, BMI-, SBP-, and smoking status-adjusted baPWV significantly increased with increasing numbers of components of MS $(1,257 \pm$ $172.2,1,371 \pm 158.0,1,438 \pm 231.5,1,648 \pm 246.8,1,669$ $\pm 344.7 \mathrm{~cm} / \mathrm{sec}, p$ for trend $<0.01$ in the new IDF criterion; $1,264 \pm 171.1,1,364 \pm 169.9,1,465 \pm 235.4,1,553 \pm$ $236.9,1,689 \pm 281.1 \mathrm{~cm} / \mathrm{sec}, p$ for trend $<0.01$ in the Japanese criterion) (Fig. 2). The subjects with 1, 2, 3, 
and 4 components of MS had significantly higher baPWV than those without MS in both of the criteria $(p<0.001$ for all). It was also shown in the two subgroups, laborers and office workers, that baPWV increased with increasing number of components of MS after controlling for age, BMI, SBP, and smoking status ( $p$ for trend $<0.01$ in both criteria for laborers; $p$ for trend $<0.05$ in both criteria for office workers) (data not shown).

To assess the utility of the two new criteria of MS to identify individuals with increased baPWV, we analyzed the risk of increased baPWV in relation to MS in the two criteria by a multiple logistic regression analysis. After controlling for age, BMI, SBP, and smoking status, the odds ratios for increased baPWV in subjects with MS were $2.07(95 \% \mathrm{CI}=0.90-4.81)$ in the Japanese criterion and 2.88 (1.30-6.36) in the new IDF criterion compared with those without MS (Table 2).

\section{Discussion}

In the present study, only $1.6 \%$ of all subjects were classified as having central obesity using the NCEP ATP III criterion of central obesity (data not shown) and the prevalence of MS by the NCEP ATP III criteria was $5.84 \%$ which is a quarter of the prevalence of $24.0 \%$ reported in the U.S. using the NCEP ATP III criteria ${ }^{40)}$. Because the NCEP ATP III criteria are based on data from Caucasian cohorts, the great difference of the prevalence of MS may reflect not only the prevalence itself but also the build between races and, thus, the NCEP ATP III criteria may not be appropriate for Japanese.

On the other hand, the prevalences of MS by the new IDF and Japanese criteria were $17.0 \%$ and $13.5 \%$, respectively, in middle-aged Japanese men. Because the new criteria use ethnic specific values for waist circumference, the difference of MS prevalence between Japan $(17.0 \%$ by the new IDF criterion; $13.5 \%$ by the new IDF criterion) and the U.S. (24.0\% by the NECP ATP III criterion) may reflect the true value of the difference of MS prevalence. Thus, the new two criteria may be better for diagnosis of MS than the NCEP ATP III criterion, although we understand that the present data cannot be simply compared with previous data obtained using the other criterion of MS.

The two new criteria are similar in that they regard central obesity as an indispensable item and include the criteria of dyslipidemia, hyperglycemia, and hypertension. However, there are two different points. First, the fasting plasma glucose threshold is higher in the Japanese criterion than in the new IDF criterion. If the cutoff value for the fasting plasma glucose in the Japanese criterion $(\geq 110 \mathrm{mg} / \mathrm{dL})$ is substituted with the cutoff value in the new IDF criterion ( $\geq 100 \mathrm{mg} / \mathrm{dL}$ ), the number of subjects with MS increases by 9 to $60(15.9 \%)$ which is almost the same number as that of the MS subjects by the new IDF criterion. Therefore, the difference in the prevalence of MS between the new IDF criterion (17.0\%) and the Japanese criterion (13.5\%) was mainly the result of a difference in the fasting plasma glucose threshold. Second, while the new IDF criterion uses two items of dyslipidemia, i.e. HDL cholesterol and triglyceride, for the criteria of MS on equal terms with other criteria, the Japanese criterion put the two items together as dyslipidemia. Weighting each trait equally in the new IDF criterion may overweight the contribution of dyslipidemia to MS, though it is unlikely that each trait confers equivalent disease risk.

Of the 377 subjects, $14(3.7 \%)$ satisfied the IDF criterion of MS but did not satisfy the Japanese criterion of MS. Their mean value of baPWV was $1,482.4 \pm 290.1$ and it was not significantly different from that of those who satisfied both criteria (baPWV=1,585.8 \pm 255.0 , $p=0.241$ ) or that of those who satisfied neither criteria (baPWV=1,362.1 $\pm 204.6, p=0.148$ ). Therefore, we could not conclude which of the two criteria was good for Japanese men in terms of a predictor for high baPWV.

The present study demonstrated that MS was significantly associated with a risk of increased baPWV after adjustment for the potential confounding factor of aortic stiffness in both the new criteria. This assures that multiple risk factors act together and exert an even greater influence on future events than expected by each risk factor alone. Our data concurs with a previous study which reported that clustered features of MS are related to increased aortic $\mathrm{PWV}^{5}$. However, in the previous study, the characteristics of MS were not defined by any proposed criteria but by their original criterion because the aim of the study was to investigate the association between clusters features of MS and increased aortic PWV.

Although the present study also demonstrated that the new IDF criterion was a better predictor of increased baPWV than the Japanese criterion, further studies are expected to assess the utility of the two new criteria to identify individuals with increased cardiovascular risk, because baPWV is a surrogate marker of cardiovascular disease.

The present study has several limitations. First, the number of subjects was relatively small and the results of this study need to be assessed in larger sample size. However, there was a strong and positive association of MS components with the level of baPWV after adjustment for potential confounding factors in both of the new criteria, and the relationship between baPWV and MS was not associated with the kind of occupation according to the analysis of the subgroups of laborers and office workers. Therefore, we suggest that the two new criteria are good for diagnosing MS among Japanese men in large populations. Second, we used a surrogate measurement of arterial stiffness and therefore may not have identified its prevalence precisely. However, previous studies have 
shown that PWV reflects vascular damage ${ }^{28-30)}$ and severity of cardiovascular disease ${ }^{31)}$, and predicts future events $^{32-34)}$; moreover, PWV is a non-invasive marker. Therefore, PWV may have been an appropriate marker for arterial stiffness in the present study. Finally, our cross-sectional study design lacks information on the time sequence of events and, thus, does not permit identification of causal relationships.

In conclusion, the new IDF criterion and the Japanese criterion are both good for diagnosing MS among Japanese men, because a linear increase in baPWV occurred with increasing MS components after adjustment for potential confounding factors in both the new criteria. Our study was a cross-sectional study, and further prospective studies are expected to follow.

Acknowledgments: This research was supported by the Ministry of Economy, Trade and Industry, Japan (R\&D for practical use of university-based technology by matching government and private funds). We thank all the staff and study participants.

\section{References}

1) GM Reaven: Banting lecture 1988: role of insulin resistance in human disease. Diabetes 37, 1595-1607 (1988)

2) NM Kaplan: The deadly quartet: upper-body obesity, glucose intolerance, hypertriglyceridemia, and hypertension. Arch Intern Med 149, 1514-1520 (1989)

3) MI Schmidt, RL Watson, BB Duncan, P Metcalf, FL Brancati, AR Sharrett, CE Davis and G Heiss: Clustering of dyslipidemia, hyperuricemia, diabetes, and hypertension and its association with fasting insulin and central and overall obesity in a general population. Metabolism 45, 699-706 (1996)

4) PWF Wilson, WB Kannel, H Silbershatz and RB D'Agostino: Clustering of metabolic factors and coronary heart disease. Arch Intern Med 159, 11041109 (1999)

5) N Nakanishi, K Suzuki and K Tatara: Clustered features of the metabolic syndrome and the risk for increased aortic pulse wave velocity in middle-aged Japanese men. Angiology 54, 551-559 (2003)

6) C Lorenzo, M Okoloise, K Williams, MP Stern and SM Haffner: San Antonio Heart Study. The metabolic syndrome as predictor of type 2 diabetes: the San Antonio heart study. Diabetes Care 26, 3153-3159 (2003)

7) N Nakanishi, T Takatorige, H Fukuda, K Shirai, W Li, M Okamoto, H Yoshida, Y Matsuo, K Suzuki and K Tatara: Components of the metabolic syndrome as predictors of cardiovascular disease and type 2 diabetes in middle-aged Japanese men. Diabetes Res Clin Pract 64, 59-70 (2004)

8) B Isomaa, P Almgren, T Tuomi, B Forsen, K Lahti, M Nissen, MR Taskinen and L Groop: Cardiovascular morbidity and mortality associated with the metabolic syndrome. Diabetes Care 24, 683-689 (2001)
9) HM Lakka, DE Laaksonen, TA Lakka, LK Niskanen, E Kumpusalo, J Tuomilehto and JT Salonen: The metabolic syndrome and total and cardiovascular disease mortality in middle-aged men. JAMA 288 , 2709-2716 (2002)

10) S Malik, ND Wong, SS Franklin, TV Kamath, GJ L'Italien, JR Pio and GR Williams: Impact of the metabolic syndrome on mortality from coronary heart disease, cardiovascular disease, and all causes in United States adults. Circulation 110, 1245-1250 (2004)

11) AM McNeill, WD Rosamond, CJ Girman, G Heiss, SH Golden, BB Duncan, HE East and C Ballantyne: Prevalence of coronary heart disease and carotid arterial thickening in patients with the metabolic syndrome (The ARIC Study). Am J Cardiol 94, 1249-1254 (2004)

12) AM McNeill, WD Rosamond, CJ Girman, SH Golden, MI Schmidt, HE East, CM Ballantyne and G Heiss: The metabolic syndrome and 11-year risk of incident cardiovascular disease in the atherosclerosis risk in communities study. Diabetes Care 28, 385-390 (2005)

13) KG Alberti and PZ Zimmet: Definition, diagnosis and classification of diabetes melli-tus and its complications: Part 1: diagnosis and classification of diabetes mellitus provisional report of a WHO consultation. Diabet Med 15, 539-553 (1998)

14) Expert Panel on Detection, Evaluation, and Treatment of High Blood Cholesterol in Adults: Executive summary of the third report of the National Cholesterol Education Program (NCEP) expert panel on detection, evaluation, and treatment of high blood cholesterol in adults (Adult Treatment Panel III). JAMA 285, 24862497 (2001)

15) YW Park, S Zhu, L Palaniappan, S Heshka, MR Carnethon and SB Heymsfield: The metabolic syndrome: prevalence and associated risk factor findings in the US population from the Third National Health and Nutrition Examination Survey, 1988-1994. Arch Intern Med 163, 427-436 (2003)

16) CE Tan, S Ma, D Wai, SK Chew and ES Tai: Can we apply the National Cholesterol Education Program Adult Treatment Panel definition of the metabolic syndrome to Asians? Diabetes Care 27, 1182-1186 (2004)

17) WY Lee, JS Park, SY Noh, EJ Rhee, SW Kim and PZ Zimmet: Prevalence of the metabolic syndrome among 40,698 Korean metropolitan subjects. Diabetes Res Clin Pract 65, 143-149 (2004)

18) ES Kim, SM Han, YI Kim, KH Song, MS Kim, WB Kim, JY Park and KU Lee: Prevalence and clinical characteristics of metabolic syndrome in a rural population of South Korea. Diabet Med 21, 1141-1143 (2004)

19) ME Jorgensen, P Bjerregaard, F Gyntelberg and K Borch-Johnsen: Greenland population study: prevalence of the metabolic syndrome among the Inuit in Greenland. A comparison between two proposed definitions. Diabet Med 21, 1237-1242 (2004)

20) R Gupta, PC Deedwania, A Gupta, S Rastogi, RB Panwar and K Kothari: Prevalence of metabolic syndrome in an Indian urban population. Int J Cardiol 
97, 257-261 (2004)

21) B Enkhmaa, K Shiwaku, E Anuurad, A Nogi, K Kitajima, M Yamasaki, T Oyunsuren and Y Yamane: Prevalence of the metabolic syndrome using the Third Report of the National Cholesterol Educational Program Expert Panel on Detection, Evaluation, and Treatment of High Blood Cholesterol in Adults (ATP III) and the modified ATP III definitions for Japanese and Mongolians. Clin Chim Acta 352, 105-113 (2005)

22) GN Thomas, SY Ho, ED Janus, KS Lam, AJ Hedley and TH Lam: Hong Kong Cardiovascular Risk Factor Prevalence Study Steering Committee: The US National Cholesterol Education Programme Adult Treatment Panel III (NCEP ATP III) prevalence of the metabolic syndrome in a Chinese population. Diabetes Res Clin Pract 67, 251-257 (2005)

23) T Tillin, N Forouhi, DG Johnston, PM McKeigue, N Chaturvedi and IF Godsland: Metabolic syndrome and coronary heart disease in South Asians, AfricanCaribbeans and white Europeans: a UK populationbased cross-sectional study. Diabetologia 48, 649-656 (2005)

24) International Diabetes Federation (IDF): The IDF consensus worldwide definition of the metabolic syndrome [article online]. Available from http:// www.idf.org/home.

25) Definition and diagnosis criteria of metabolic syndrome. Nippon Naika Gakkai Zasshi 94, 794-809 (2005) (in Japanese)

26) R Asmar, A Benetos, J Topouchian, P Laurent, B Pannier, AM Brisac, R Target, and BI Levy: Assessment of arterial distensibility by automatic pulse wave velocity measurement: validation and clinical application studies. Hypertension 26, 485-490 (1995)

27) ED Lehmann: Clinical value of aortic pulse-wave velocity measurement. Lancet 354, 528-529 (1999)

28) U Nakamura, M Iwase, S Nohara, H Kanai, K Ichikawa and M Iida: Usefulness of brachial-ankle pulse wave velocity measurement: correlation with abdominal aortic calcification. Hypertens Res 26, 163-167 (2003)

29) A Yamashina, H Tomiyama, T Arai, K Hirose, Y Koji, Y Hirayama, Y Yamamoto and S Hori: Brachial-ankle pulse wave velocity as a marker of atherosclerotic vascular damage and cardiovascular risk. Hypertens Res 26, 615-622 (2003)

30) M Sawabe, R Takahashi, S Matsushita, T Ozawa, T Arai, A Hamamatsu, K Nakahara, K Chida, H Yamanouchi, S Murayama and N Tanaka: Aortic pulse wave velocity and the degree of atherosclerosis in the elderly: a pathological study based on 304 autopsy cases. Atherosclerosis 179, 345-351 (2005)

31) HE Lim, CG Park, SH Shin, JC Ahn, HS Seo and DJ Oh: Aortic pulse wave velocity as an independent marker of coronary artery disease. Blood Press 13, 369375 (2004)

32) J Blacher, AP Guerin, B Pannier, SJ Marchais, ME Safar and GM London: Impact of aortic stiffness on survival in end-stage renal disease. Circulation 99, 2434-1439 (1999)

33) S Laurent, P Boutouyrie, R Asmar, I Gautier, B Laloux, L Guize, P Ducimetiere and A Benetos: Aortic stiffness is an independent predictor of all-cause and cardiovascular mortality in hypertensive patients. Hypertension 37, 1236-1241 (2001)

34) T Shokawa, M Imazu, H Yamamoto, M Toyofuku, $N$ Tasaki, T Okimoto, K Yamane and N Kohno: Pulse wave velocity predicts cardiovascular mortality: findings from the Hawaii-Los Angeles-Hiroshima study. Circ J 69, 259-264 (2005)

35) A Yamashina, H Tomiyama, K Takeda, H Tsuda, T Arai, K Hirose, Y Koji, S Hori and Y Yamamoto: Validity, reproducibility, and clinical significance of noninvasive brachial-ankle pulse wave velocity measurement. Hypertens Res 25, 359-364 (2002)

36) R Imanishi, S Seto, G Toda, M Yoshida, A Ohtsuru, $Y$ Koide, T Baba and K Yano: High brachial-ankle pulse wave velocity is an independent predictor of the presence of coronary artery disease in men. Hypertens Res 27, 71-78 (2004)

37) S Sakuragi, J Iwasaki, N Tokunaga, S Hiramatsu and T Ohe: Aortic stiffness is an independent predictor of left ventricular function in patients with coronary heart disease. Cardiology 103, 107-112 (2005)

38) J Amar, JB Ruidavets, B Chamontin, L Drouet and J Ferrieres: Arterial stiffness and cardiovascular risk factors in a population-based study. J Hypertens 19, 381-387 (2001)

39) KM Choi, KW Lee, JA Seo, JH Oh, SG Kim, NH Kim, DS Choi and SH Baik: Relationship between brachialankle pulse wave velocity and cardiovascular risk factors of the metabolic syndrome. Diabetes Res Clin Pract 66, 57-61 (2004)

40) ES Ford, WH Giles and WH Dietz: Prevalence of the metabolic syndrome among US adults: findings from the third National Health and Nutrition Examination Survey. JAMA 287, 356-359 (2002) 